\title{
Median Arcuate Ligament syndrome: Case Report
}

\author{
Kumar Vaibhav, Soumya Khanna, Satyendra Tiwary, Puneet and Ajay K Khanna \\ Institute of Medical Sciences, Banaras Hindu University, Varanasi-221005, India
}

\section{Article Info}

*Corresponding author:
Ajay K Khanna
Professor of Surgery
Institute of Medical Sciences
Banaras Hindu University
Varanasi 221005, India
E-mail: akhannabhu@gmail.com

Received: May 31, 2019

Accepted: August 13, 2019

Published: August 21, 2019

Citation: Vaibhav K, Khanna S, Tiwary S, Puneet, Khanna AK. Median Arcuate Ligament syndrome: Case Report. Madridge J Surg. 2019; 2(1): 72-74.

doi: $10.18689 / \mathrm{mjs}-1000117$

Copyright: (c) 2019 The Author(s). This work is licensed under a Creative Commons Attribution 4.0 International License, which permits unrestricted use, distribution, and reproduction in any medium, provided the original work is properly cited.

Published by Madridge Publishers

\begin{abstract}
Median arcuate ligament syndrome is a rare disorder that is clinically characterized by the triad of postprandial abdominal pain, weight loss, and often an abdominal bruit due to compression of the celiac artery by the median arcuate ligament. The treatment of this condition is to release the coeliac axis by division of median arcuate ligament. We encountered this patient of Median Arcuate ligament Syndrome in almost 40 years of clinical practice so it is an extremely rare condition.
\end{abstract}

Keywords: Diaphragm; Epigastric pain; Coeliac axis; Compression; Vascular.

\section{Introduction}

The median arcuate ligament is a muscular and fibrous arch under the diaphragm where the right and left crus of the diaphragm connect together. This ligament passes superior to the origin of the celiac artery and continues to the posterior diaphragm, encompasses the aorta, at the level of the first lumbar vertebral body, superior to the origin of the celiac axis.

However, in $10 \%-24 \%$ of people, the ligament may be low and therefore cross over the proximal portion of the celiac axis, causing a characteristic indentation. In a small subset of these patients, the median arcuate ligament can compress the proximal celiac axis enough to be hemodynamically significant and cause symptoms [1].

Median arcuate ligament syndrome (MALS) or celiac artery compression syndrome was first described by Harjola [2] in 1963 and Dunbar et al. [3] in 1965 is a rare disorder characterized by the compression of the celiac trunk by the median arcuate ligament of the diaphragm, compromising the blood flow.

\section{Case Report}

A 35 years old male presented to hospital with complains of pain abdomen since 6 months, mainly epigastric, dull aching, aggravated postprandial and associated with nausea. There was no history of weight loss. On examination faint bruit was heard in epigastrium without any other sign in abdomen. Patient was referred to the radiology department for abdominal sonogram including mesenteric Doppler.

Real-time color and spectral Doppler examination of the abdominal aorta and its major branches (celiac trunk and superior mesenteric artery) was interrogated in the supine and erect position after eight hours of fasting. The results indicated stenosis of the celiac artery (Figure 1) with turbulent flow with increased peak systolic velocity and post stenotic dilatation. Further Contrast enhanced tomography angiography of abdomen was done which showed focal short segment narrowing of proximal celiac trunk and hooked appearance due to indentation by median arcuate ligament (Figure 2). Mild post stenotic dilatation of artery was noted with no collaterals. The patient was operated and release of median arcuate ligament and celiac trunk was done. Post 
operative no complications and patient followed regularly on outpatient basis.

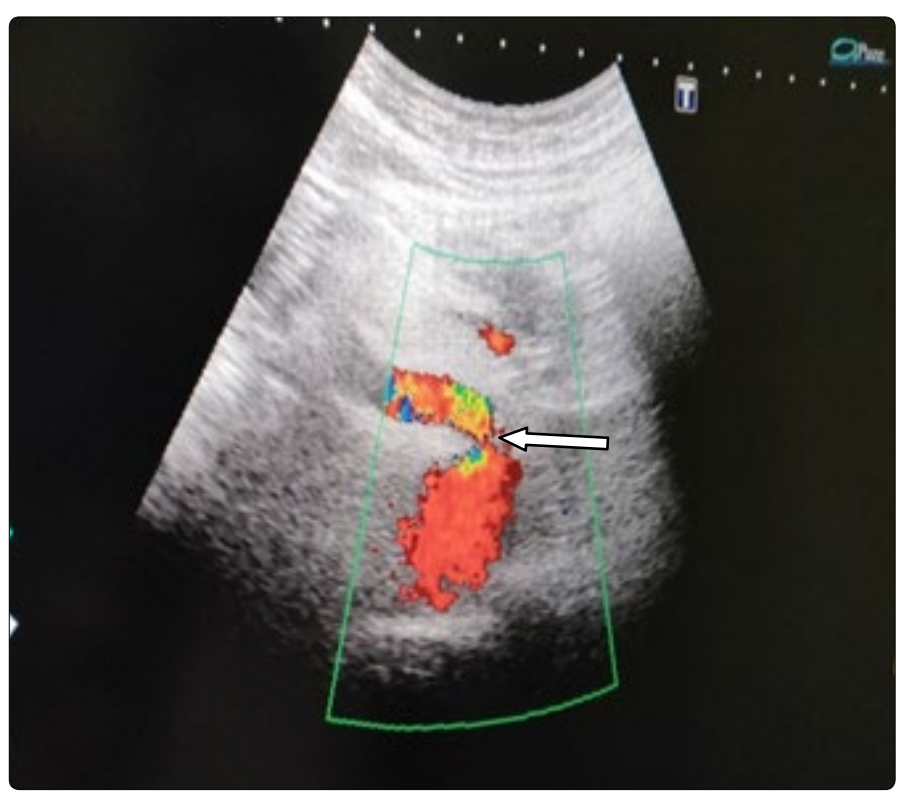

Figure 1. Arrow showing stenosis of Celiac trunk with post stenotic dilataion.

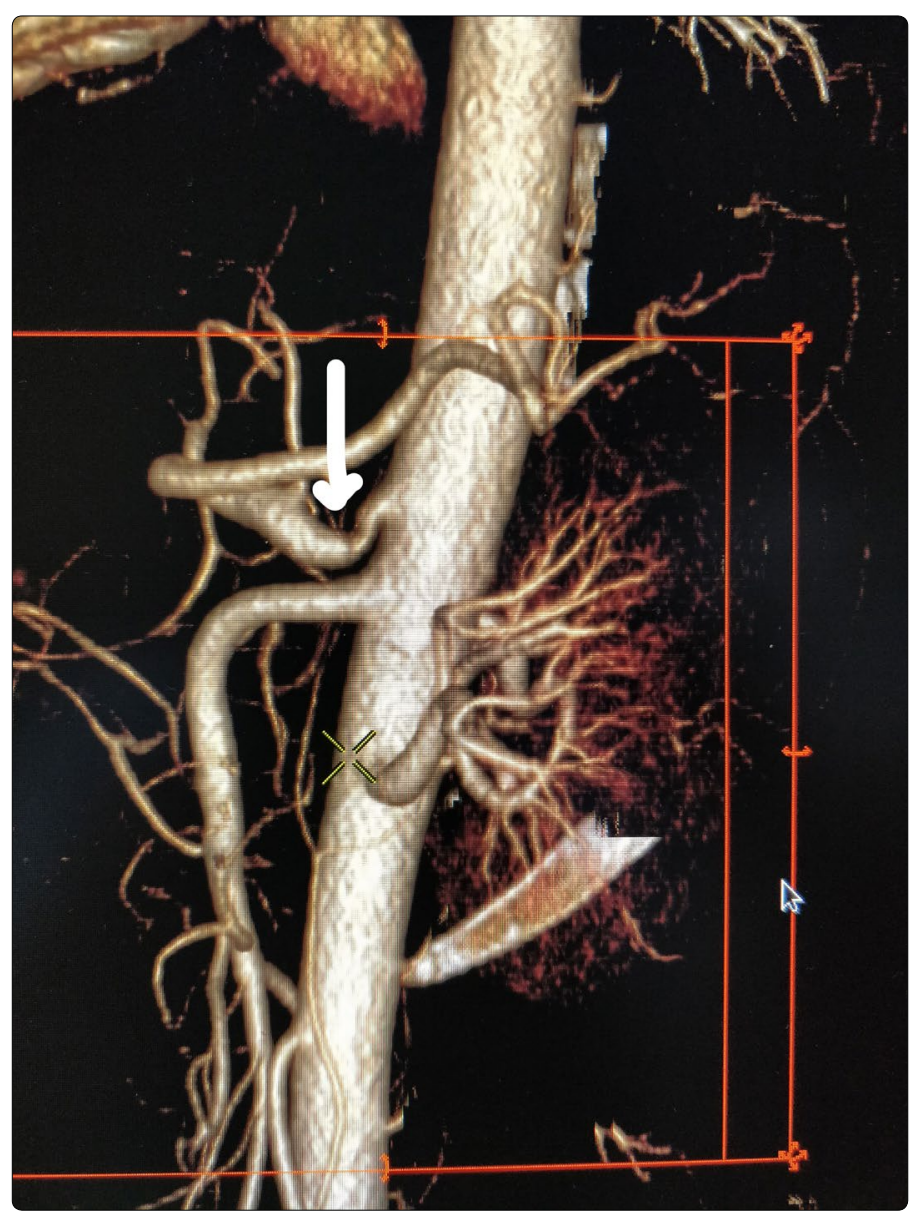

Figure 2. Sagittal 3D image demonstrates acute angulation and narrowing of the proximal celiac axis. There is minimal post stenotic dilatation, with "hooked" appearance (arrow) that is characteristic of median arcuate ligament syndrome.

\section{Discussion}

MALS is a condition that is seldom considered a differential diagnosis for abdominal pain in daily practice.
Although the incidence of MAL syndrome in the population is not well known, it is more prevalent in women (4:1 ratio) between the ages of 30 to 50 years and in patients with a thin body habitus [4]. Patients present with postprandial epigastric pain, nausea and vomiting, and subsequently weight loss (typically over 20 pounds) [5]. Other symptoms may include diarrhea and delayed gastric emptying, and in some individuals, an abdominal bruit may be heard. It is vital to note the difference between median arcuate ligament syndromes from median arcuate ligament compression. Median arcuate ligament compression occurs in $10 \%$ to $25 \%$ of the population and does not cause any symptoms [3]. Only about $1 \%$ of these individuals who have severe compression experience symptoms. The pain from MALS is believed to be caused by the median arcuate ligament compressing on the plexus of nerve fibers surrounding the celiac trunk. When suspicious for this syndrome, Doppler sonography may be performed to measure the blood flow through the proximal celiac artery during inspiration and expiration. In a normal celiac artery, uniform velocities and waveform configurations (without turbulence) would be present. Peak systolic velocities higher than normal represent stenosis and turbulent flow. Additional noninvasive imaging studies to aid in the diagnosis of MAL syndrome include CT angiography and magnetic resonance angiography. Computed tomography angiography offers the advantage of 3-dimensional reconstruction and allows visualization of the compressed artery from different angles. Treatment of MALS is aimed at restoring the normal blood flow of the celiac artery and eliminating the irritation caused by the celiac nerve fibers. Decompression of the celiac artery is the general approach for treatment. The preferred management strategy is by surgically or laparoscopically dividing or separating the median arcuate ligament in combination with destruction or removal of the celiac ganglion. If these interventions are unsuccessful, endovascular methods such as per cutaneous transluminal angioplasty may be another alternative for treatment [6].

Unfortunately, the diagnosis of MALS is usually made after more common conditions have been ruled out. In the meantime, the patient may endure months to years of recurrent abdominal pain without knowledge of etiology. For this reason, in patients with upper gastrointestinal disorders, especially postprandial pain that persists after medical therapy, it may be useful to perform vascular investigation sooner with duplex sonography to evaluate the possibility of median arcuate ligament syndrome.

\section{References}

1. Lindner $\mathrm{HH}$, Kemprud E. A clinicoanatomic study of the arcuate ligament of the diaphragm. Arch Surg. 1971; 103(5): 600-605. doi: 10.1001/archsurg.1971.01350110102016

2. Harjola PT. A rare obstruction of the coeliac artery; report of case. Ann Chir Gynaecol Fenn. 1963; 52: 547-550.

3. Dunbar JD, Molnar W, Beman FF, Marable, SA. Compression of the celiac trunk and abdominal angina. Am J Roentgenol Radium Ther Nucl Med.1965; 95(3): 731-744. 
4. Trinidad-Hernandez M, Keith P, Habib I, White JV. Reversible gastroparesis: functional documentation of celiac axis compression syndrome and postoperative improvement. Am Surg. 2006; 72(4): 339-344.

5. Cleveland Clinic. Median arcuate ligament syndrome (MALS). Accessed July 23. 2018.
6. Duffy AJ, Panait L, Eisenberg D, Bell RL, Roberts KE, Sumpio B. Management of median arcuate ligament syndrome: a new paradigm. Ann Vasc Surg. 2009; 23(6): 778-84. doi: 10.1016/j.avsg.2008.11.005 